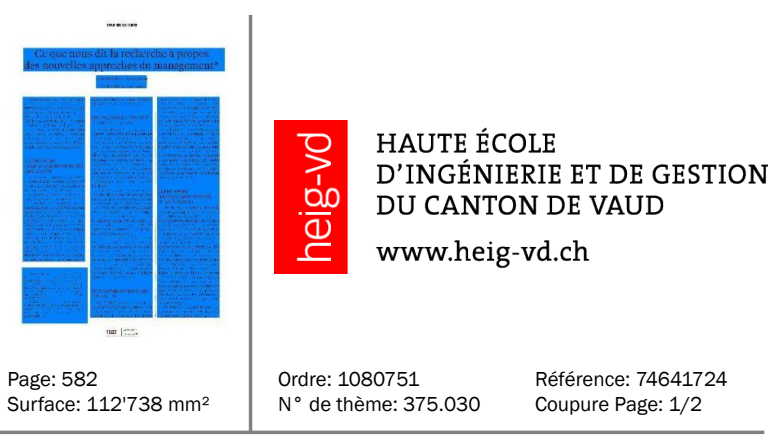

\title{
Ce que nous dit la recherche à propos des nouvelles approches du management*
}

CÉLINE DESMARAIS Et JEAN WEIDMANN

Rev Med Suisse 2019; 15: 1582-3

Nombreux sont les acteurs du monde de la santé qui aspirent à développer des pratiques plus agiles, plus respectueuses des personnes et des patients ou clients, et plus satisfaisantes pour les collaborateurs. Mais le fatalisme règne face à ces enjeux complexes: est-il possible de promouvoir un management collectif et démocratique dans le secteur des soins? La recherche s'intéresse aux moteurs des comportements humains et analyse les effets des modèles démocratiques d'organisation.

\section{DES EXPÉRIENCES EMBLÉMATIQUES ET INSPIRANTES DANS LA SANTÉ}

Il existe des expériences qui ont su concilier efficacité économique, bien-être au travail et qualité des soins. L'entreprise néerlandaise Buurtzorg de soins à domicile a montré que rompre avec la taylorisation ${ }^{a}$ galopante des soins à domicile était possible, en s'appuyant sur des équipes qualifiées et autonomes et sur une organisation agile et légère. Les rapports d'audit menés par Ernst et Young en 2010 et KPMG en 2015 ont entériné la compatibilité entre satisfaction des patients et des collaborateurs et résultats économiques. Dans le domaine hospitalier, face à la pénurie croissante de personnels soignants, des chercheurs ont observé que certains hôpitaux demeuraient attractifs: ils ont nommé hôpitaux magnétiques ${ }^{2}$ des établissements qui s'appuient sur l'autonomie professionnelle, la valorisation du leadership infirmier, un climat relationnel collégial entre médecins et soignants.

\section{DES APPROCHES QUI DEPASSENT LE DOMAINE DE LA SANTÉ}

Les expériences d'organisations promouvant l'autonomie des collaborateurs ne sont pas propres à la santé. A la suite de précurseurs comme la FAVI en France ou Gore Tex aux Etats-Unis, ${ }^{3}$ de nombreuses organisations ont expérimenté des hiérarchies plates, des décisions collectives et un partage du pouvoir. Réaction à un management toujours plus contrôlant ou adaptation à un monde volatile, incertain, complexe et ambigu (selon le célèbre acronyme VUCA), ces expériences ont été modélisées par des consultants et des chercheurs.

L'holacratie, issue des recherches sur la sociocratie, est une méthode qui organise le travail à partir de cercles (et non plus d'équipes) qui remplissent différents rôles. Les décisions y sont prises de façon collective (lorsqu'il n'y a plus d'objection). Les membres du cercle n'ont plus de cahier des charges prescrit, mais une liste de rôles qui changent selon les besoins de l'environnement, ce qui permet une meilleure réactivité.

Le concept d'entreprise libérée décrit des organisations dans lesquelles on considère que ceux qui font savent ce qu'il y a à faire et qu'il n'est donc pas nécessaire de les contrôler. Enfin, les organisations dites «opales», ${ }^{6}$ obéissent à des principes similaires et considèrent l'organisation comme un être vivant qui doit valoriser l'autonomie des individus pour s'adapter rapidement à l'environnement.

\section{DES FONDEMENTS THÉORIQUES DÉJÀ ANCIENS}

Ces modèles peuvent sembler disruptifs. Leurs fondements théoriques n'ont pourtant rien de nouveau. Dans les années soixante déjà, le mouvement des relations humaines contestait l'efficacité de la rationalisation et du contrôle promus par les modèles tayloriens. Douglas Mac Grégor ${ }^{7}$ a par ailleurs montré que les croyances des dirigeants ont des conséquences tangibles: si ceux-ci pensent que leurs collaborateurs sont désireux de bien faire leur travail, ils mettront en place des organisations fondées sur la confiance et l'autonomie et les employés se sentiront en conséquence engagés et responsables. L'entreprise libérée part de ce même principe: l'homme est bon! Les travaux sur l'autodétermination ${ }^{8}$ montrent également à quel point l'engagement des salariés repose sur leur sentiment d'avoir prise sur leur travail, ce qui suppose de favoriser l'autonomie pour disposer de collaborateurs engagés et créatifs.

\section{UNE RECHERCHE \\ SUR CES NOUVEAUX MODÈLES \\ EN SUISSE ROMANDE}

Afin de mieux comprendre les conditions de développement et les effets de ces modèles, une équipe de la HEIG-VD ${ }^{9}$ a mené récemment une recherche en Suisse romande auprès d'entreprises ayant transformé leurs pratiques. Celle-ci confirme les avantages de ces nouvelles façons de travailler: engagement accru, plus grande satisfaction au travail, créativité, développement de nouvelles compétences. Elle confirme par ailleurs ce que les critiques de ces modèles annoncent souvent : en mettant en avant la responsabilité de chacun, on augmente les risques de stress et de sur-engagement. Elle montre enfin que chaque organisation invente un cheminement qui lui est propre, mais qu'il existe des conditions communes du succès: poser un cadre précis, mettre en place une 
Revue Médicale Suisse 1225 Chêne-Bourg 022/7029311 https://www.revmed.ch/
Genre de média: Médias imprimés Type de média: Presse spécialisée Tirage: 4'914 Parution: 43x/année

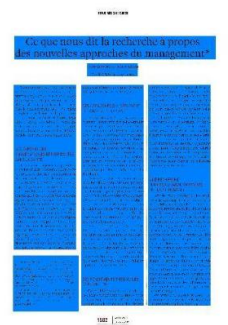

Page: 582 Surface: $112^{\prime} 738 \mathrm{~mm}^{2}$
HAUTE ÉCOLE

D'INGÉNIERIE ET DE GESTION DU CANTON DE VAUD

www.heig-vd.ch approche incrémentale du changement en consultant largement les collaborateurs, développer la confiance, changer la posture des managers...

On attribue au marquis de Condorcet la formule «conservons par la sagesse ce quenousavons acquis parl'enthousiasme!». Celle-ci se prête bien aux expériences de leadership démocratique: elles doivent être portées par une conviction forte dans la nécessité de développer l'humain et doivent se construire de manière pragmatique et concertée!

1 Monsen K, de Blok J.Buurtzorg Nederland. Am J Nursing 2013;113:55-9.

2 Brunelle Y. Les hôpitaux magnétiques: un hôpital où il fait bon travailler en est un où il fait bon se faire soigner. Pratiques et Organisation des Soins 2009;40:39

$3 \mathrm{https}: / /$ changethework.com/gore-tex-management/ 4 Robertson B. Holacracy: The revolutionary management system that abolishes hierarchy. London: Penguin UK, 2015.

5 Getz I, Carney B, Demange $O$. Quand la liberté des salariés fait le succès des entreprises. Paris: Flammarion coll. «Champs essais, 2013.

6 Laloux F. Reinventing Organizations : Vers des communautés de travail inspirées. Paris: Diateino, 2015.

7 McGregor D. Theory $X$ and theory $Y$. Organization theory 1960;358:374.

8 Ryan RM, Deci EL. Self-determination theory and the facilitation of intrinsic motivation, social development and well-being. Am Psychol 2000;55:68.

9 Weidmann J, Konishi M, Gonin F, Agassiz I. Power

sharing within organizations: Benefits, drawbacks and

conditions. An empirical study on Holacracy and related methods for self-managed teams. European Academy of Management (EURAM), Lisbon, Portugal, 2019.

\section{PRS CÉLINE DESMARAIS ET JEAN WEIDMANN}

Haute Ecole d'Ingénierie et de Gestion du Canton de Vaud (HEIG-VD)

Avenue des Sports 20, 1401 Yverdon-les-Bains

University of Applied Sciences and Arts Western

Switzerland (HES-SO)

celine.desmarais@heig-vd.ch

jean.weidmann@heig-vd.ch
* Cet article est le résumé d’une présentation effectuée lors de la journée d'échanges sur le thème «Mutation dans le management des organisations de santé», le 4 juin 2019 au Centre de Congrès Beaulieu, Lausanne. Organisation: Haute Ecole d'Ingénierie et de Gestion du Canton de Vaud, en partenariat avec le Réseau Santé Nord Broye, Arsanté et l'Institut et Haute Ecole de la Santé La Source.

${ }^{a}$ La taylorisation fait référence au modèle d'organisation du travail élaboré par W. Taylor qui consiste à séparer la conception et l'exécution du travail, découper les tâches et les spécialiser à l'extrême, formaliser et contrôler les processus de travail. 